\title{
ARTICLES
}

\section{Derivation of the energy-time uncertainty relation}

\author{
Donald H. Kobe and V. C. Aguilera-Navarro* \\ Department of Physics, University of North Texas, Denton, Texas 76203-5368
}

(Received 28 June 1993)

\begin{abstract}
A derivation from first principles is given of the energy-time uncertainty relation in quantum mechanics. A canonical transformation is made in classical mechanics to a new canonical momentum, which is energy $E$, and a new canonical coordinate $T$, which is called tempus, conjugate to the energy. Tempus $T$, the canonical coordinate conjugate to the energy, is conceptually different from the time $t$ in which the system evolves. The Poisson bracket is a canonical invariant, so that energy and tempus satisfy the same Poisson bracket as do $p$ and $q$. When the system is quantized, we find the energy-time uncertainty relation $\Delta E \Delta T \geq \hbar / 2$. For a conservative system the average of the tempus operator $\hat{T}$ is the time $t$ plus a constant. For a free particle and a particle acted on by a constant force, the tempus operators are constructed explicitly, and the energy-time uncertainty relation is explicitly verified.
\end{abstract}

PACS number(s): 03.65.Bz, 03.20. $+\mathrm{i}$

\section{INTRODUCTION}

Ever since the discovery of quantum mechanics, the energy-time uncertainty relation $\Delta E \Delta t \geq \hbar / 2$ has had a different basis than the position-momentum uncertainty relation $\Delta q \Delta p \geq \hbar / 2$. In nonrelativistic quantum mechanics time $t$ is a parameter, not an operator as is the coordinate $q$. Therefore the usual quantum-mechanical average and uncertainty in the time cannot be calculated as in the case of the coordinate. For this reason, it has been suggested that the energy-time uncertainty relation simply be eliminated from quantum mechanics [1]. This radical suggestion overlooks the many different approaches to the physically useful energy-time uncertainty relation.

We briefly mention some of the derivations of the energy-time uncertainty relation, even though some are only heuristic $[2,3]$. (i) The energy-time uncertainty relation can be obtained by showing that a wave packet of width $\Delta t$ in time requires a spread in angular frequencies $\Delta \omega$ such that $\Delta \omega \Delta t \sim 1$. Together with the Planck relation $E=\hbar \omega$, we obtain $\Delta E \Delta t \sim \hbar$ [4]. (ii) The energytime uncertainty relation is derived by reducing it to the position-momentum uncertainty relation $[3,5]$. If $\Delta E=(\partial E / \partial p) \Delta p=v \Delta p$ and $\Delta t=\Delta q / v$, where $v$ is the group velocity, then $\Delta E \Delta t=\Delta p \Delta q \geq \hbar / 2$. (iii) Mandelstam and Tamm [6] showed that the energy-time uncertainty relation can be derived from the Heisenberg equation of motion for an arbitrary operator $\hat{A}$ and the generalized uncertainty relation for $\hat{A}$ and the Hamiltonian $\hat{H}[3,7]$. If we define $\Delta t$ to be the smallest $\Delta t_{A} \equiv \Delta A /(d\langle\hat{A}\rangle / d t)$ for all operators $\hat{A}$, and

*Permanent address: Instituto de Física Teórica, UNESP, 01405-000 São Paulo, São Paulo, Brazil.
$\Delta E=\Delta H$, we also recover the energy-time uncertainty relation [8,9]. (iv) Wigner [10] has considered the expectation value of $t^{n}$ in the state $\psi=\psi(q, t)$ to be

$$
\left\langle t^{n}\right\rangle=\frac{\int_{-\infty}^{\infty} d t t^{n}|\psi(q, t)|^{2}}{\int_{-\infty}^{\infty} d t|\psi(q, t)|^{2}} .
$$

Therefore he defines the square of the uncertainty $(\Delta t)^{2}=\left\langle t^{2}\right\rangle-\langle t\rangle^{2}$ at constant $q$ and defines $(\Delta E)^{2}$ similarly. He shows that $\Delta E \Delta t \geq \hbar / 2$ holds at constant $q$. Cook [11] uses this form of the uncertainty relation to analyze the thought experiment that Einstein [12] proposed to Bohr at the 1930 Solvay Conference. (v) Many authors [13-29] have tried to find a time operator $\hat{t}$, which satisfies the canonical commutation relation $[\hat{t}, \hat{H}]=i \hbar$. Then the energy-time uncertainty relation can be derived by the same procedure as for the position-momentum uncertainty relation [7]. These attempts have to contend with Pauli's argument [30] that a time operator cannot exist in general because the spectrum of the time operator is continuous and unbounded, while the spectrum of the Hamiltonian may be discrete and is bounded from below. The role between the time $t$ as a parameter and the time operator $\hat{t}$ is often not clear in these attempts. A clear distinction is however made by Razavy $[17,18]$. (vi) In scattering theory it is the time of arrival which is important and attempts have been made to find an operator which describes this quantity [31-36]. (vii) In the context of the density matrix $\rho$, Eberly and Singh [37] have shown that $\partial \rho / \partial t$ plays the role of an "inverse time operator" from which the energy-time uncertainty relation can be derived [38]. (viii) The role of the uncertainty relations in relativistic quantum theory has been discussed by a number of authors [39-43]. (ix) The interpretation of the energy-time uncertainty relation is a part of quantum measurement theory [44-51] and is distinct 
from its derivation. The energy-time uncertainty relation for decaying states has been improved by Gislason, Sabelli, and Wood [52].

In this paper we give a derivation of the energy-time uncertainty relation, which is based on first principles and does not resort to any heuristic or ad hoc arguments. Our derivation is firmly based on nonrelativistic classical mechanics and its quantization, and puts the energy-time uncertainty relation on the same footing as the positionmomentum uncertainty relation. In classical mechanics we make a canonical transformation [53] from the old canonical variables $(q, p)$ to new canonical variables $\left(q^{\prime}, p^{\prime}\right)$, where the new canonical momentum $p^{\prime}$ is the energy $E$ of the particle and the new canonical coordinate $q^{\prime}$ is a quantity $T$ conjugate to the energy, which we call tempus [54-56]. This tempus $T$ has dimension of time, but is conceptually different from the time $t$ in which the system evolves. Since $T$ and $E$ are canonically conjugate variables and the Poisson bracket is a canonical invariant [53], their Poisson bracket is unity. Upon quantization, the tempus operator $\hat{T}$ and the energy operator $\hat{E}$ satisfy the canonical commutation relation. Therefore, the energy and the tempus operators satisfy the usual energy-time uncertainty relation $\Delta E \Delta T \geq \hbar / 2$ as long as the expectation values of $\hat{T}$ and $\hat{T}^{2}$ exist. For a conservative system the expectation value of the tempus operator is equal to the time $t$ plus an irrelevant constant. The distinction between the tempus operator $\hat{T}$ conjugate to the energy operator and the time $t$ of evolution is thus made clear.

This approach is applied to two examples: (i) a free particle and (ii) a particle acted on by a constant force. In both cases the tempus operator $\hat{T}$ is constructed explicitly by quantizing the corresponding classical tempus $T$ conjugate to the energy. In the subspace of the Hilbert space in which it exists, the average value $\langle\hat{T}\rangle=t$ plus a constant. The energy-time uncertainty relation is explicitly shown to be satisfied.

In Sec. II, a canonical transformation is made in classical mechanics to energy and its canonical conjugate tempus. Using Poisson brackets, we quantize the system in Sec. III and derive the energy-time uncertainty relation. In Sec. IV, examples of a free particle and a particle acted on by a constant force are considered and it is shown that the energy-time uncertainty relation is explicitly satisfied. Finally, the conclusion is given in Sec. V.

\section{ENERGY AND TEMPUS AS CANONICAL VARIABLES}

In the Hamiltonian formulation of classical mechanics, the canonical coordinate $q$ and the canonical momentum $p$ conjugate to it satisfy Hamilton's equations

$$
\dot{q}=\frac{\partial H}{\partial p}, \dot{p}=\frac{-\partial H}{\partial q},
$$

where $H=H(q, p, t)$ is the Hamiltonian for a system of one degree of freedom and the overdot denotes the total time derivative. Using a generating function $S$, we can make a canonical transformation to a new set of canonical variables $\left(q^{\prime}, p^{\prime}\right)$, which also satisfy Hamilton's equations [54]

$$
\dot{q}^{\prime}=\frac{\partial H^{\prime}}{\partial p^{\prime}}, \quad \dot{p}^{\prime}=\frac{-\partial H^{\prime}}{\partial q^{\prime}},
$$

where the new Hamiltonian is $H^{\prime}=H+\partial S / \partial t$.

If the new canonical momentum $p^{\prime}$ is chosen to be the energy $E$, the new canonical coordinate $q^{\prime}$ conjugate to it is called tempus and denoted as $T$. This tempus canonical coordinate conjugate to the energy is conceptually different from the time $t$ in which the system evolves and is a function of $q, E$, and $t$ [55] with the dimension of time.

The generating function of the second type $S(q, E, t)$, which implements this canonical transformation, satisfies $[5,56]$,

$$
p=\frac{\partial S(q, E, t)}{\partial q},
$$

and the new canonical coordinate tempus is

$$
T=\frac{\partial S(q, E, t)}{\partial E} .
$$

By integrating Eq. (4), we obtain

$$
S(q, E, t)=\int_{q_{0}}^{q} p(\bar{q}, E, t) d \bar{q}+S\left(q_{0}, E, t\right),
$$

where $q_{0}$ is an arbitrary initial displacement. The arbitrary function $S\left(q_{0}, E, t\right)$ of $E$ and $t$ in Eq. (6) can often be chosen to be zero without loss of generality. To obtain the generating function in Eq. (6), it is necessary to find the canonical momentum $p$ as function of $q, E$, and $t$. The new generalized coordinate tempus $T$ can be obtained as a function of $q, E$, and $t$ from Eq. (5). When this equation is solved for $q$ as a function of $T, E$, and $t$, we have the solution to the problem if we know $T$ and $E$ as functions of time $t$.

The new Hamiltonian $H^{\prime}$ in Eq. (3) for the new canonical variables $\left(q^{\prime}, p^{\prime}\right)=(T, E)$ is

$$
H^{\prime}=H+(\partial S / \partial t)_{q, E} .
$$

The energy $E$ may not be equal to the Hamiltonian $H$, so their difference is defined as

$$
\Phi=H-E \text {. }
$$

When Eqs. (7) and (8) are used in Hamilton's equations (3) for the new canonical variables $(T, E)$, we obtain [55]

$$
\dot{T}=1+\left[\frac{\partial\left(\Phi+\frac{\partial S}{\partial t}\right)}{\partial E}\right]_{T, t}
$$

and

$$
\dot{E}=-\left[\frac{\partial\left(\Phi+\frac{\partial S}{\partial t}\right)}{\partial T}\right]_{E, t} .
$$

In Eqs. (9) and (10) it is necessary to express $\Phi+(\partial S / \partial t)_{q, E}$ as a function of $E, T$, and $t$ before differentiation. Hamilton's equations (9) and (10) can be solved for $T(t)$ and $E(t)$. The solution to the original problem is $q=q(E(t), T(t), t) \equiv q(t)$. 
For a conservative system, there is no explicit time dependence in $S$, so $\partial S / \partial t=0$. In this case, the Hamiltonian $H$ can be chosen to be the energy $E$, so $\Phi=H-E=0$. Therefore, Eqs. (9) and (10) reduce to

$$
\dot{T}=1, \dot{E}=0 \text {. }
$$

The solution to Eq. (11) is $T=t-t_{0}$ and $E=E_{0}$, where $t_{0}$ and $E_{0}$ are constants. In this case, tempus is equal to the time $t$ minus a constant. The solution to the original problem is therefore $q=q(E, T)=q\left(E_{0}, t-t_{0}\right)$.

\section{QUANTIZATION}

In order to quantize a system, it is necessary to replace the Poisson bracket of two functions of the canonical variables by $(i \hbar)^{-1}$ times the commutator of the corresponding operators. The Poisson bracket between $A=A(q, p, t)$ and $B=B(q, p, t)$ is defined as

$$
\{A, B\}_{q, p}=\frac{\partial A}{\partial q} \frac{\partial B}{\partial p}-\frac{\partial B}{\partial q} \frac{\partial A}{\partial p} .
$$

The Poisson bracket is a canonical invariant [53], so

$$
\{A, B\}_{q, p}=\{A, B\}_{q^{\prime}, p^{\prime}},
$$

where the new canonical variables $\left(q^{\prime}, p^{\prime}\right)$ are related to the old canonical variables $(q, p)$ by a canonical transformation. If $A=q$ and $B=p$, we have

$1=\{q, p\}_{q, p}=\left\{q^{\prime}, p^{\prime}\right\}_{q^{\prime}, p^{\prime}}=\left\{q^{\prime}, p^{\prime}\right\}_{q, p}=\{T, E\}_{q, p}$

from Eqs. (12) and (13).

When the system is quantized, the operators $\hat{q}$ and $\hat{p}$ ( $\hat{T}$ and $\hat{E}$ ) corresponding to $q$ and $p(T$ and $E$ ) satisfy the commutation relations

$$
[\hat{q}, \hat{p}]=i \hbar
$$

and

$$
[\hat{T}, \hat{E}]=i \hbar
$$

from Eq. (14).

In general, the uncertainty relation for the operators $\hat{A}$ and $\hat{B}$ in the state $\psi$ is [57-61]:

$$
\Delta A \Delta B \geq \frac{1}{2}|\langle\hat{C}\rangle| \text {, }
$$

where $i \hat{C}=[\hat{A}, \hat{B}]$ and $\langle\hat{C}\rangle \equiv\langle\psi \mid \hat{C} \psi\rangle$. The square of the uncertainty in $\hat{A}$ is defined as

$$
(\Delta A)^{2}=\left\langle\psi \mid(\hat{A}-\langle\hat{A}\rangle)^{2} \psi\right\rangle \text {. }
$$

For the energy and tempus operators in Eq. (16) we have the uncertainty relation

$$
\Delta E \Delta T \geq \hbar / 2 \text {, }
$$

where the state $\psi$ must be in the domain of $\hat{E}, \hat{E}^{2}, \hat{T}$, and $\hat{T}^{2}$. The choice of the new canonical momentum $p^{\prime}=E$ and the new canonical coordinate $q^{\prime}=T$ puts the energytime uncertainty relation in Eq. (19) on the same solid basis as the momentum-position uncertainty relation $\Delta p \Delta q \geq \hbar / 2$ [57]. In contrast to $t$, the new canonical tempus operator $\hat{T}$ is conjugate to the energy operator $\hat{E}$. The uncertainty in the tempus operator has a well defined meaning for states $\psi$ that are in the domain of $\hat{T}$ and $\hat{T}^{2}$.

For a conservative system the average of the tempus operator $\hat{T}$ is the time $t$ plus an irrelevant constant. The Schrödinger equation is

$$
\hat{H}(q, \hat{p}) \psi(q, t)=i \hbar \partial \psi(q, t) / \partial t,
$$

for a system with a time-independent Hamiltonian $\hat{H}=H(q, \hat{p})$. The time-dependent wave function $\psi(q, t)$ can be written as

$$
\psi(q, t)=U(t) \psi(q, 0),
$$

where $\psi(q, 0)$ is the wave function at time zero. The time evolution operator is

$$
U(t)=\exp [-i \hat{H} t / \hbar],
$$

where $\hat{H}$ is the time-independent Hamiltonian operator.

From the commutation relation in Eq. (16) and $\hat{E}=\hat{H}$ we can show that the expectation value of the tempus operator $\hat{T}$ is

$$
\langle\psi(t) \mid \hat{T} \psi(t)\rangle=t+\langle\psi(0) \mid \hat{T} \psi(0)\rangle,
$$

where $\langle\psi(0) \mid \hat{T} \psi(0)\rangle$ is a constant depending on the initial wave function. Therefore, Eq. (23) shows that the expectation value of the tempus operator is directly related to the time $t$ of evolution of the system.

\section{EXAMPLES}

In this section, we explicitly construct the tempus operators for (i) a free particle and (ii) a particle acted on by a constant force and show that the energy-time uncertainty relation is satisfied.

\section{A. Free particle}

The tempus operator $\hat{T}$ for a free particle is constructed from the corresponding classical expression and the energy-time uncertainty relation is obtained.

For a free particle of mass $m$, the energy $E$ in terms of the canonical momentum $p$ is

$$
E=p^{2} / 2 m \text {. }
$$

Equation (24) can be solved for the canonical momentum $p=(2 m E)^{1 / 2} \operatorname{sgn}\left(p_{0}\right)$, where the sign of the initial momentum $p_{0}$ is $\operatorname{sgn}\left(p_{0}\right)$. The generating function in Eq. (6) is

$$
S(q, E, t)=(2 m E)^{1 / 2}\left(q-q_{0}\right) \operatorname{sgn}\left(p_{0}\right),
$$

where we take the arbitrary function $S\left(q_{0}, E, t\right)=0$. The new canonical coordinate tempus is obtained from Eq. (5) and is

$$
T=(m / 2 E)^{1 / 2}\left(q-q_{0}\right) \operatorname{sgn}\left(p_{0}\right) .
$$

In this way we have derived the canonical coordinate tempus conjugate to the energy, which is conceptually distinct from the time $t$ in which the system evolves.

The solution to the classical problem is obtained by solving Eq. (26) for $q$ and using the solutions of Eq. (11). We therefore obtain

$$
q(t)=q_{0}+v_{0}\left(t-t_{0}\right),
$$


where the initial velocity $v_{0}=p_{0} / m=\left(2 E_{0} / m\right)^{1 / 2} \operatorname{sgn}\left(p_{0}\right)$ and $T=t-t_{0}$.

In order to construct a self-adjoint tempus operator, Eq. (26) can be expressed in terms of $p$ by substituting the energy in Eq. (24) into it. Then we obtain the canonical tempus coordinate for $q_{0}=0$ to be

$$
T=m q / p .
$$

Equation (28) may be quantized to obtain the tempus operator $\hat{T}$ by replacing $q$ and $p$ by their corresponding operators $\hat{q}$ and $\hat{p}$ and writing the operator in a symmetric way $[14-16,19,25]$ :

$$
\widehat{T}=\frac{1}{2} m\left(\hat{q}_{\hat{p}}^{-1}+\hat{p}^{-1} \hat{q}\right) .
$$

The domain of $\hat{T}$ is not the whole Hilbert space because of the presence of the operator $\hat{p}^{-1}$. Nevertheless, for the states in its domain, the operator $\hat{T}$ has useful properties. The argument of Pauli [30] applies only to Hermitian operators defined on the whole Hilbert space. From the commutation relation in Eq. (15) for $\hat{q}$ and $\hat{p}$, we can derive for $\hat{T}$ in Eq. (29) and the operator $\hat{E}$ corresponding to Eq. (24) the commutation relation in Eq. (16).

The uncertainty relation in Eq. (19) for energy and time can be obtained if we choose a wave function which is in the domain of $\hat{E}, \hat{E}^{2}, \hat{T}$, and $\hat{T}^{2}$. Since the tempus operator in Eq. (29) involves $\hat{p}^{-1}$, it is more convenient to work in momentum space where the realization of the commutation relation in Eq. (15) is $\hat{p}=p$ and $\hat{q}=i \hbar \partial / \partial p$. We choose a wave function in momentum space at time $t=0$ to be

$$
\phi(p, 0)=N p^{n} \exp \left(-a_{0} p^{2}\right),
$$

where $a_{0}$ is a real positive constant, $n$ is an arbitrary integer $\geq 2$, and $N$ is the normalization constant. Since the Schrödinger equation in momentum space is

$$
\hat{H}(\widehat{q}, p, t) \phi(p, t)=i \hbar \partial \phi(p, t) / \partial t,
$$

the solution at time $t \neq 0$ with the Hamiltonian $\hat{H}$ equal to the energy $E$ in Eq. (24) is

$$
\phi(p, t)=N p^{n} \exp \left(-a p^{2}\right),
$$

where $a=a_{0}+i t / 2 m \hbar$ is a complex function of time. With the wave function in Eq. (32), the average of the tempus operator $\hat{T}$ in Eq. (29) is

$$
\langle\phi \mid \hat{T} \phi\rangle=\langle\hat{T}\rangle=t,
$$

the time $t$ in which the system evolves, as expected from Eq. (23).

The square of the uncertainty in the tempus operator $\widehat{T}$ at time $t$ is

$$
\begin{aligned}
(\Delta T)^{2} & \equiv\left\langle\phi \mid(\hat{T}-\langle\hat{T}\rangle)^{2} \phi\right\rangle \\
& =\left\langle\hat{T}^{2}\right\rangle-\langle\hat{T}\rangle^{2} \\
& =\left(2 a_{0} m \hbar\right)^{2} /\left(n-\frac{3}{2}\right) .
\end{aligned}
$$

The square of the uncertainty in the energy operator $\hat{E}$ at time $t$ is

$$
\begin{aligned}
(\Delta E)^{2} & \equiv\left\langle\phi \mid(\hat{E}-\langle\hat{E}\rangle)^{2} \phi\right\rangle \\
& =\left\langle\hat{E}^{2}\right\rangle-\langle\hat{E}\rangle^{2} \\
& =(n+1 / 2)\left(4 m a_{0}\right)^{-2}
\end{aligned}
$$

From Eqs. (34) and (35) the product of $\Delta E$ and $\Delta T$ is $\Delta E \Delta T=[1+2 /(n-3 / 2)]^{1 / 2} \hbar / 2 \geq \hbar / 2$ for $n \geq 2$,

so the uncertainty relation is established.

As $n \rightarrow \infty$, Eq. (36) shows that $\Delta E \Delta T=\hbar / 2$, i.e., equality holds in the uncertainty relation Eq. (36). The reason equality holds is that in the limit as $n \rightarrow \infty$, there is no distinction between $n+4, n+3, \ldots, n-2$. Then we have the following:

(i) as $n \rightarrow \infty$

$$
(\hat{T}-\langle\hat{T}\rangle) \phi=\gamma(\hat{E}-\langle\hat{E}\rangle) \phi,
$$

where $\gamma$ is a constant, and

(ii)

$$
\begin{array}{r}
\langle[(\hat{T}-\langle\hat{T}\rangle)(\hat{E}-\langle\hat{E}\rangle)+(\hat{E}-\langle\hat{E}\rangle)(\hat{T}-\langle\hat{T}\rangle)]\rangle \\
=2 \operatorname{Re}\langle(\hat{T}-\langle\hat{T}\rangle) \phi \mid(\hat{E}-\langle\hat{E}\rangle) \phi\rangle=0,
\end{array}
$$

which are the two conditions for the equality to hold in the uncertainty relation [57].

\section{B. Particle acted on by a constant force}

For a particle acted on by a constant force $F_{0}$, the Hamiltonian $H$ is

$$
H=p^{2} / 2 m-F_{0} q=E,
$$

which is also the energy $E$. Equation (39) can be solved for the canonical momentum $p$,

$$
p=(2 m)^{1 / 2}\left(E+F_{0} q\right)^{1 / 2} .
$$

When Eq. (40) is substituted into Eq. (6) for the generating function $S$, and the integral is performed, we obtain

$S(q, E, t)=\left(2 / 3 F_{0}\right)(2 m)^{1 / 2}\left[\left(E+F_{0} q\right)^{3 / 2}-\left(E+F_{0} q_{0}\right)^{3 / 2}\right]$

$$
+S\left(q_{0}, E, t\right) \text {. }
$$

The canonical coordinate tempus $T$ conjugate to the energy is obtained from Eq. (5), which gives

$$
\begin{aligned}
T(q, E, t)=F_{0}^{-1}(2 m)^{1 / 2} & {\left[\left(E+F_{0} q\right)^{1 / 2}\right.} \\
& \left.-\left(E+F_{0} q_{0}\right)^{1 / 2}\right],
\end{aligned}
$$

where $T\left(q_{0}, E, t\right)=0$. Since the Hamiltonian $H$ in Eq. (39) is conservative, Hamilton's equations (11) have the solution $T=t-t_{0}$ and $E=E_{0}$, where $t_{0}$ and $E_{0}$ are constants. If Eq. (42) is solved for $q$, we obtain

$$
q(t)=q_{0}+v_{0}\left(t-t_{0}\right)+(1 / 2) a\left(t-t_{0}\right)^{2},
$$

where $q_{0}=q\left(t_{0}\right)$ is the initial position, $v_{0}=\dot{q}\left(t_{0}\right)$ is the initial velocity, and $a=\ddot{q}\left(t_{0}\right)=F_{0} / m$ is the constant acceleration. Equation (43) is the solution to the classical problem. 
In order to obtain a Hermitian tempus operator $\hat{T}$, it is necessary to express tempus in Eq. (42) in terms of $q$ and p. When Eq. (39) for the energy is substituted into Eq. (42) for the canonical coordinate tempus we obtain $T=p / F_{0}$ for $p_{0}=0$. When this expression is quantized, we obtain [23]

$$
\hat{T}=\hat{p} / F_{0},
$$

where $\hat{p}$ is the canonical momentum operator. Because the potential energy in Eq. (39) is not bounded from below, Pauli's argument [30] for the nonexistence of a Hermitian time operator does not apply to this example.

By a direct calculation we can show that the tempus operator $\hat{T}$ in Eq. (44) and the energy operator $\hat{E}$ corresponding to Eq. (39) satisfy the canonical commutation relation in Eq. (16). Therefore, from Eq. (17) the energytime uncertainty relation in Eq. (19) is also satisfied.

\section{CONCLUSION}

In this paper a derivation of the energy-time uncertainty relation is given based on first principles. It is not necessary to make use of any ad hoc or heuristic assumptions. A classical canonical transformation is made from the old canonical variables $(q, p)$ to new canonical variables $\left(q^{\prime}, p^{\prime}\right)$, where the new canonical momentum $p^{\prime}$ is chosen to be the energy $E$ and the new canonical coordi- nate $q^{\prime}$ conjugate to the energy is called tempus $T$. This tempus canonical coordinate has the dimension of time, but is conceptually different from the time $t$ in which the system evolves. In general, tempus $T$ is a function of $q$, $E$, and $t$. The energy $E$ and tempus $T$ have the same Poisson bracket as do $p$ and $q$, since the Poisson bracket is a canonical invariant. When the system is quantized, the operators $\hat{E}$ and $\hat{T}$ satisfy the same commutation relations as do the operators $\hat{p}$ and $\hat{q}$. Therefore, $\hat{E}$ and $\hat{T}$ satisfy the same uncertainty relation as do $\hat{p}$ and $\hat{q}$. The energy-time uncertainty relation is therefore put on the same basis as the momentum-position uncertainty relation. The approach used here to derive the energy-time uncertainty relation has a firm basis in classical mechanics, which makes a clear distinction between the time of evolution $t$ and the canonical coordinate tempus $T$ conjugate to the energy. For a conservative classical system, tempus $T$ is equal to the time $t$ plus a constant. For a conservative quantum system, the average of the tempus operator $\hat{T}$ is equal to the time $t$ plus a constant.

\section{ACKNOWLEDGMENTS}

We would like to thank Dr. Henry A. Warchall, Jr. for discussions concerning some mathematical aspects of the paper. V.C.A.-N. acknowledges financial support from FAPESP, Brazil.
[1] M. Bunge, Can. J. Phys. 48, 1410 (1970).

[2] V. V. Dodonov and V. I. Man'ko, in Invariants and the Evolution of Nonstationary Quantum Systems, edited by M. A. Markov (Nova Science, Commack, NY, 1989), pp. 3-101.

[3] H. C. Ohanian, Principles of Quantum Mechanics (Prentice-Hall, Englewood Cliffs, NJ, 1990), pp. 133-138.

[4] D. B. Beard and G. B. Beard, Quantum Mechanics with Applications (Allyn and Bacon, Boston, 1970), pp. 19-21.

[5] C. Cohen-Tannoudji, B. Diu, and F. Laloë, Quantum Mechanics (Wiley, New York, 1977), Vol. 1, p. 252.

[6] L. Mandelstam and Ig. Tamm, J. Phys. (Moscow) 9, 249 (1945).

[7] A. Messiah, Quantum Mechanics (North-Holland, Amsterdam, 1961), Vol. I, pp. 129-139 and pp. 319 and 320.

[8] M. Grabowski, Lett. Math. Phys. 8, 455 (1984).

[9] K. Bhattacharyya, J. Phys. A 16, 2993 (1983).

[10] E. P. Wigner, in Aspects of Quantum Theory, edited by A. Salam and E. P. Wigner (Cambridge, London, 1972), pp. 237-247.

[11] L. F. Cook, Am. J. Phys. 48, 142 (1980).

[12] N. Bohr, in Albert Einstein: Philosopher-Scientist, edited by P. A. Schilpp (Open Court, LaSalle, IL, 1970), pp. 201-241.

[13] F. Engelmann and E. Fick, Nuovo Cimento Suppl. 12, 63 (1959).

[14] H. Paul, Ann. Phys. (Leipzig) 9, 252 (1962).

[15] E. Fick and F. Engelmann, Z. Phys. 175, 271 (1963).

[16] F. Engelmann and E. Fick, Z. Phys. 178, 551 (1964).

[17] M. Razavy, Am. J. Phys. 35, 955 (1967).

[18] M. Razavy, Nuovo Cimento B 63, 271 (1969).

[19] D. M.Rosenbaum, J. Math. Phys. 10, 1127 (1969).
[20] M. Bauer and P. A. Mello, Ann. Phys. (N.Y.) 111, 38 (1978).

[21] M. Bauer, Ann. Phys. (N.Y.) 150, 1 (1983).

[22] I. Fujiwara, Prog. Theor. Phys. 44, 1701 (1970).

[23] I. Fujiwara, Prog. Theor. Phys. 64, 18 (1980).

[24] I. Fujiwara, K. Wakita, and H. Yoro, Prog. Theor. Phys. 64, 363 (1980).

[25] T. Goto, K. Yamaguchi, and N. Sudo, Prog. Theor. Phys. 66, 1525 (1981).

[26] T. Goto, S. Naka, and K. Yamaguchi, Prog. Theor. Phys. 66, 1915 (1981).

[27] E. Recami, in The Uncertainty Principle and Foundations of Quantum Mechanics, edited by W. C. Price and S. S. Chissick (Wiley, New York, 1977), pp. 21-28.

[28] V. S. Olkhovsky, E. Recami, and A. J. Gerasimchuk, Nuovo Cimento A 22, 263 (1974).

[29] E. Papp, Int. J. Theor. Phys. 10, 385 (1974).

[30] W. Pauli, Die allgemeinen Prinzipien der Wellenmechanik, edited by S. Flügge, Encyclopedia of Physics (SpringerVerlag, Berlin, 1958), Vol. V, pt. 1, pp. 1-168. See especially footnote 1 on p. 60 .

[31] F. T. Smith, Phys. Rev. 118, 349 (1960).

[32] B. A. Lippmann, Phys. Rev. 151, 1023 (1966).

[33] G. R. Allcock, Ann. Phys. (N.Y.) 53, 253 (1969).

[34] G. R. Allcock, Ann. Phys. (N.Y.) 53, 286 (1969).

[35] G. R. Allcock, Ann. Phys. (N.Y.) 53, 311 (1969).

[36] M. Razavy, Can. J. Phys. 49, 3075 (1971).

[37] J. H. Eberly and L. P. S. Singh, Phys. Rev. D 7, 359 (1973).

[38] C. H. Blanchard, Am. J. Phys. 50, 642 (1982).

[39] E. Prugovecki, Found. Phys. 12, 555 (1982).

[40] R. Arshansky and L. P. Horwitz, Found. Phys. 15, 701 
(1985).

[41] Y. S. Kim and M. E. Noz, Found. Phys. 9, 375 (1979).

[42] D. Han, M. E. Noz, Y. S. Kim, and D. Son, Phys. Rev. D 27, 3032 (1983).

[43] P. E. Hussar, Y. S. Kim, and M. E. Noz, Am. J. Phys. 53, 142 (1985).

[44] V. Fock and N. Krylov, J. Phys. (Moscow) 11, 112 (1947).

[45] Y. Aharonov and D. Bohm, Phys. Rev. 122, 1649 (1961).

[46] V. A. Fock, Zh. Eksp. Teor. Fiz. 42, 1135 (1962) [Sov. Phys. JETP 15, 784 (1962)].

[47] Y. Aharonov and D. Bohm, Phys. Rev. B 134, 1417 (1964).

[48] Y. Aharonov and J. L. Sajko, Ann. Phys. (N.Y.) 91, 279 (1975).

[49] Yu. I. Vorontsov, Usp. Fiz. Nauk. 133, 351 (1981) [Sov. Phys. Usp. 24, 150 (1981)].

[50] M. Moshinsky, Am. J. Phys. 44, 1037 (1976).

[51] J. Rayski and J. M. Rayski, Jr., in The Uncertainty Relation and Foundations of Quantum Mechanics, edited by W. C. Price and S. S. Chissick (Wiley, New York, 1977), pp. 13-20.

[52] E. A. Gislason, N. H. Sabelli, and J. W. Wood, Phys. Rev.
A 31, 2078 (1985).

[53] H. Goldstein, Classical Mechanics, 2nd ed. (AddisonWesley, Reading, MA, 1980), pp. 378-401.

[54] D. H. Kobe, Am. J. Phys. 56, 252 (1988).

[55] D. H. Kobe, Am. J. Phys. 61, 1031 (1993).

[56] D. H. Kobe, Eur. J. Phys. 14, 262 (1993).

[57] E. Merzbacher, Quantum Mechanics, 2nd ed. (Wiley, New York, 1970), pp. 158-161.

[58] A. S. Davydov, Quantum Mechanics, 2nd ed. (Pergamon, New York, 1976), pp. 36 and 37.

[59] H. P. Robertson, Phys. Rev. 34, 163 (1929); 35, 667 (1930).

[60] See, e.g., J. von Neumann, Mathematical Foundations of Quantum Mechanics (Princeton University Press, Princeton, NJ, 1955), pp. 230-247. He gives a rigorous derivation of the position-momentum uncertainty relation in Hilbert space. Footnote 131 gives some historical remarks.

[61] P.-O. Löwdin, in Advances in Quantum Chemistry, edited by P.-O. Löwdin (Academic, New York, 1987), Vol. 3, pp. 323-381. See especially pp. 336-337 and p. 345. 